\title{
Calculation of light transistor structural parameters based on InGaN - AIGaN heterostructure
}

\author{
Artem Bushuev*, Artem Zolotov, and Ruslan Chkalov \\ Vladimir State University, 87 Gorky Street, Vladimir 600000, Russia
}

\begin{abstract}
The article is devoted to study of building possibility of digital conversion systems based on new optoelectronic principles, which allow to expand the frequency range of conversion, as well as to increase the conversion capacity, thereby increasing the accuracy. The structural parameters of emitting $\mathrm{p}-\mathrm{n}$ junction for a light-emitting transistor based on $\mathrm{p}-\mathrm{InGaN}-\mathrm{n}-\mathrm{AlGaN}$ heterostructure have been investigated.
\end{abstract}

\section{Introduction}

LEDs and emitters based on quantum dots are not yet capable of high-speed performance due to design features. Developments are underway to create light transistors that have a number of advantages over LEDs: high temperature stability (no thermostating is required), due to which there are practically no crystal lattice vibrations, low current consumption (due to the amplifying properties of the transistor), etc. [1-4].

\section{Calculation of light transistor structural parameters based on heterostructure $\mathrm{p}-\mathrm{In}_{0.06} \mathrm{Ga}_{0.94} \mathrm{~N}-\mathrm{n}-\mathrm{Al}_{0.15} \mathrm{Ga}_{0.85} \mathrm{~N}$}

The structure chosen for simulation is a bipolar light-emitting transistor based on p-InGaN $\mathrm{n}$-AlGaN, containing a quantum well between the base and the emitter [5]. Structural scheme of light transistor layers is shown in Fig. 1.

Using the software package for modeling semiconductor structures, which is part of the Comsol Multiphysics 5.3a design tool, the active double heterojunction region, from which light is emitted, was simulated. The emitting region consists of an $\operatorname{In}_{0.06} \mathrm{Ga}_{0.94} \mathrm{~N}$ layer $50 \mathrm{~nm}$ thick and two $\mathrm{Al}_{0.15} \mathrm{Ga}_{0.85} \mathrm{~N}$ layers $0.15 \mu \mathrm{m}$ thick. Since this emitter is laterally invariant, it can be modeled using a 1D line cut in the z-direction [6]. The cross-sectional area of the device is calculated based on the fact that the active area has lateral dimensions of 200x200 $\mu \mathrm{m}$.

\footnotetext{
*Corresponding author: artem_bushuev_1996@mail.ru
} 


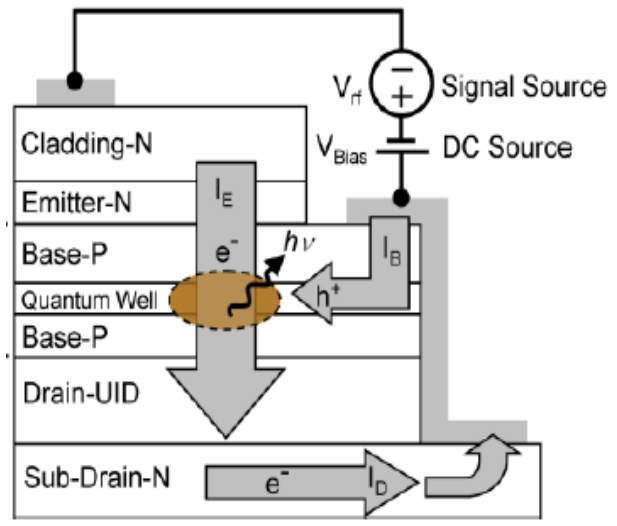

Fig. 1. Structural scheme of the light transistor layers [5].

Analysis of this structure should begin with plotting the doping depth graph and transition from a continuous heterojunction model at the quasi-Fermi level to a more suitable thermal emission model. This research has two phases. In the first stage, when $3.3 \mathrm{~V}$ is applied to the p-region of the device, forward bias reduces the height of potential barrier between n-type and p-type regions. As seen in Fig. 2, the energy level of quasi-Fermi electrons is above the edge of the conduction band in InGaN layer, so it is expected that the conduction band will be filled with electrons in this region. The energy level of quasi-Fermi holes is very close to the edge of the valence band, so the valence band will be filled with holes [7].

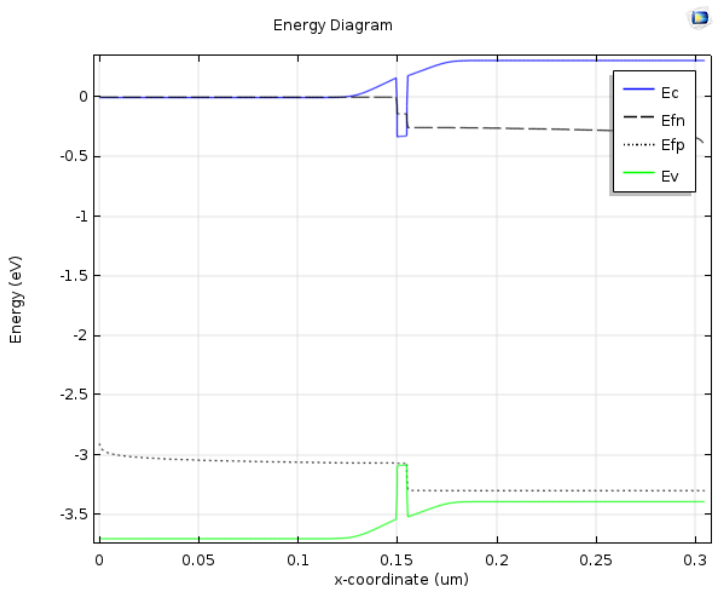

Fig. 2. Energy levels of heterojunction $\operatorname{In}_{0.06} \mathrm{Ga}_{0.94} \mathrm{~N}-\mathrm{Al}_{0.15} \mathrm{Ga}_{0.85} \mathrm{~N}$ at an applied voltage of $3.3 \mathrm{~V}$ : Ec - conduction band energy level, Efn - quasi-Fermi electrons energy level, Efp - quasi-Fermi holes energy level, Ev - valence band energy level.

The next step is to simulate the concentration of charge carriers in the structure at a forward bias of 3.3 V. As expected, when forward bias is applied, it forces carriers to collect in a potential well created by the $\operatorname{In}_{0.06} \mathrm{Ga}_{0.94} \mathrm{~N}$ layer. It is important to note that the concentration of electrons in the conduction band is similar to the concentration of holes in the valence band, as shown in Fig. 3. This makes it possible to provide an effective light flux, since each emitted photon requires the recombination of an electron through the passband with a hole [8]. 


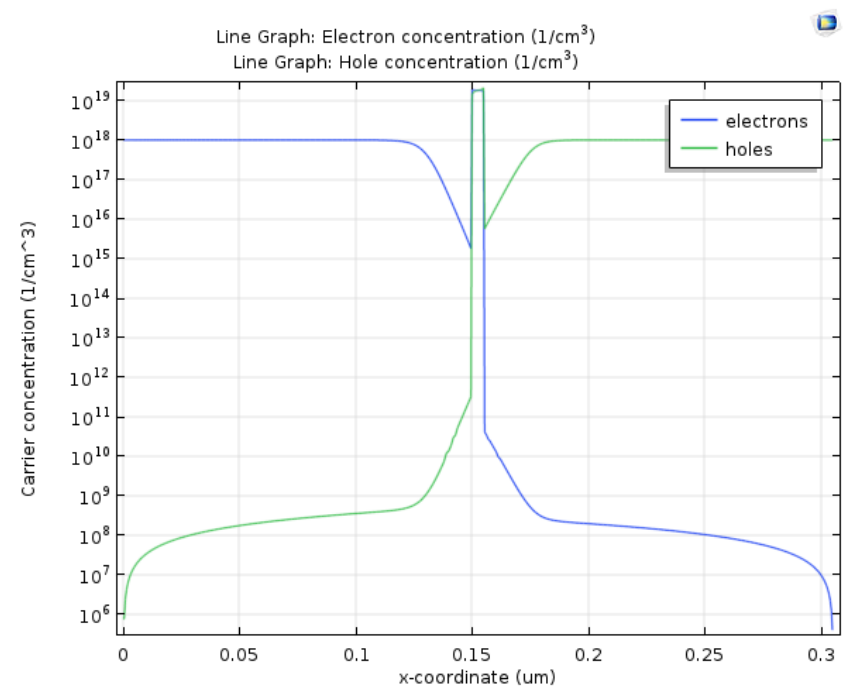

Fig. 3. Charge carrier concentration plot for the $\operatorname{In}_{0.06} \mathrm{Ga} 0.94 \mathrm{~N}-\mathrm{Al}_{0.15} \mathrm{Ga} 0.85 \mathrm{~N}$ heterostructure at a forward bias voltage of $3.3 \mathrm{~V}$.

The emission spectrum from the $\mathrm{In}_{0.06} \mathrm{Ga}_{0.94} \mathrm{~N}$ layer is shown in Fig. 4. Peak emission occurs in the photon energy range $2.8-2.9 \mathrm{eV}$, which corresponds to the wavelength range of $\sim 430-445 \mathrm{~nm}$.

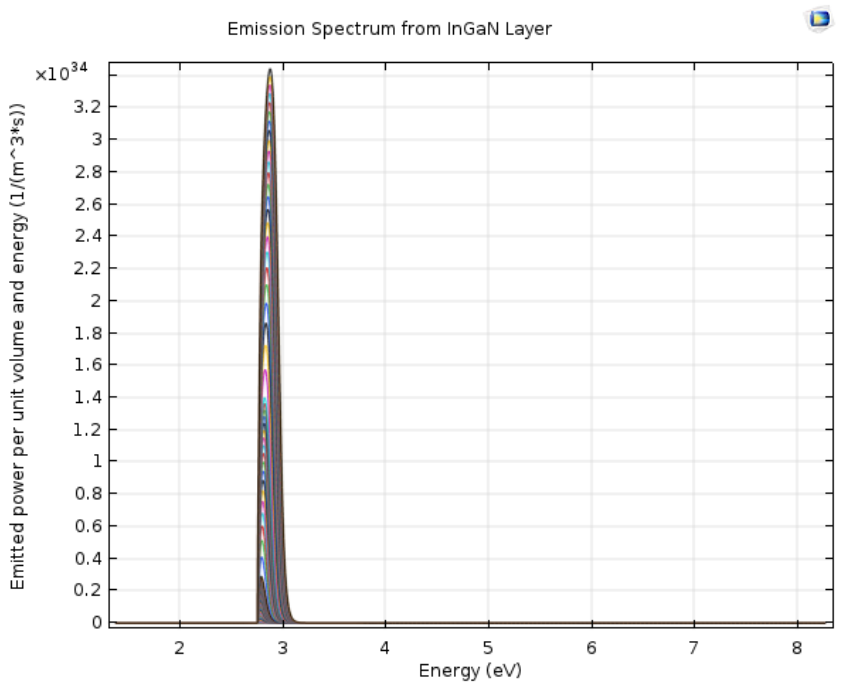

Fig. 4. The plot of radiation energy dependence on photon energy at a forward bias voltage of $3.3 \mathrm{~V}$.

According to the Fig. 5, with an increase in current value, the total radiation intensity increases. It should be noted that it is not always advantageous to increase the amount of light emitted by increasing the current. This is due to the fact that the efficiency of device decreases with increasing current density, which leads to a sublinear increase in the total radiation rate with current bias [7,9], which is confirmed by Fig. 6 . 


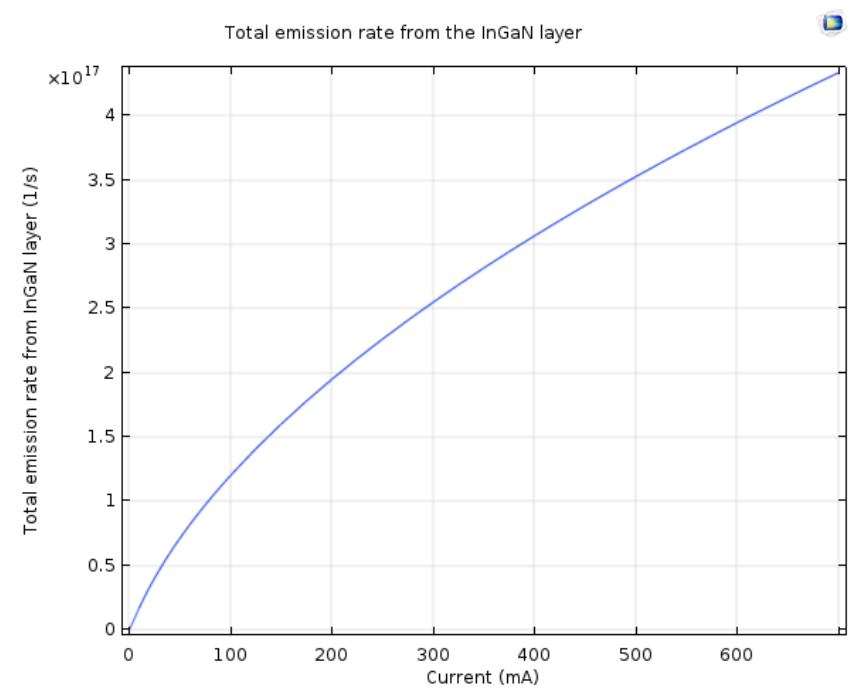

Fig. 5. Dependence of radiation intensity on applied current.

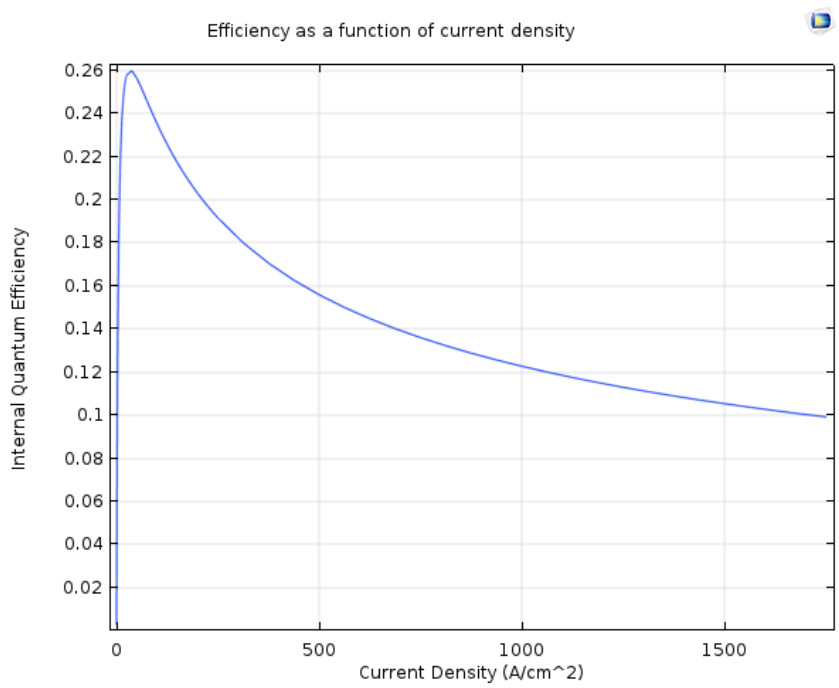

Fig. 6. The plot of dependence of radiation intensity on current density.

According to Fig. 6, there is a peak in the radiation intensity at a current density of about $30 \mathrm{~A} / \mathrm{cm}^{2}$, at a current density above $100 \mathrm{~A} / \mathrm{cm}^{2}$, a drop in intensity is observed. Maximum efficiency is achieved when currents are applied in the range of 10-30 $\mathrm{mA}$, which is typical for light-emitting devices of this size [10-12].

The current-voltage characteristic illustrated in Fig. 7 shows that the structure under study can emit at sufficiently high current values, however, according to Fig. 6, the radiation intensity imposes a limitation on the potential maximum value if the device needs to be operated with a high efficiency value. 


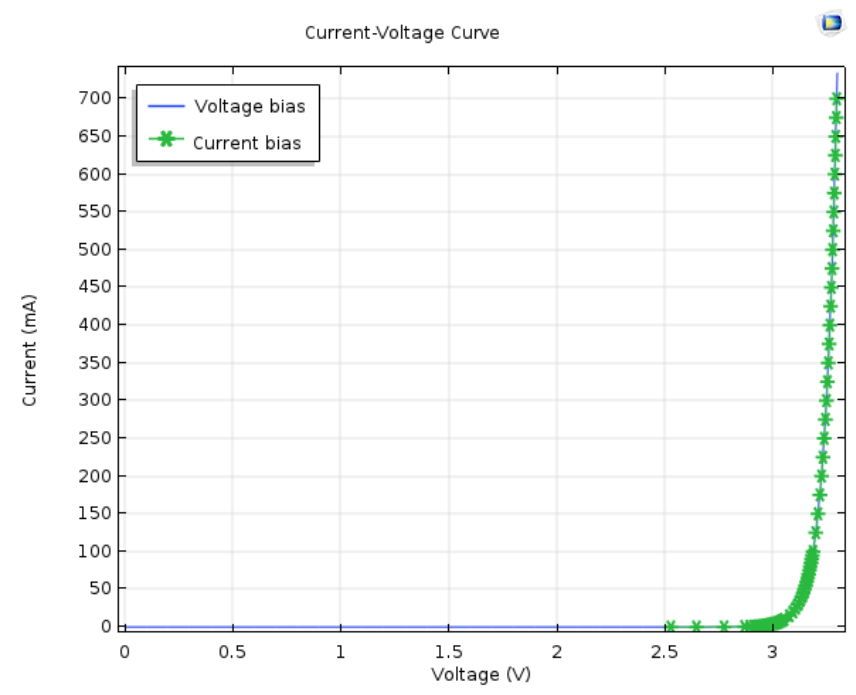

Fig. 7. Current-voltage characteristic of $\operatorname{In}_{0.06} \mathrm{Ga} 0.94 \mathrm{~N}-\mathrm{Al}_{0.15} \mathrm{Ga} 0.85 \mathrm{~N}$ heterostructure.

\section{Conclusion}

A model of an emitting transition of a light transistor with a crystal size of 200x $200 \mu \mathrm{m}$ is considered, the emitting region of which consists of one $\operatorname{In}_{0.06} \mathrm{Ga}_{0.94} \mathrm{~N}$ layer $50 \mathrm{~nm}$ thick and two $\mathrm{Al}_{0.15} \mathrm{Ga}_{0.85} \mathrm{~N}$ layers $0.15 \mu \mathrm{m}$ thick. Based on the calculation results, it was found that with an applied forward voltage of $3.3 \mathrm{~V}$, maximum efficiency is achieved when currents are applied in the range of 10-30 mA, which is typical for light-emitting devices of this size. There is a peak in the radiation intensity at a current density of about $30 \mathrm{~A} / \mathrm{cm}^{2}$, a drop in intensity is observed at a current density above $100 \mathrm{~A} / \mathrm{cm}^{2}$.

\section{References}

1. R. Windisch, A. Knobloch, J. Potemans, Light-emitting diodes with $17 \%$ external quantum efficiency at $622 \mathrm{Mb} / \mathrm{s}$ for high-bandwidth parallel short-distance optical interconnects, IEEE Journal of Selected Topics in Quantum Electronics, 5, pp. 166-171 (1999)

2. M. Akbulut, C. Chen, M. Hargis, Digital communications above $1 \mathrm{~Gb} / \mathrm{s}$ using 890-nm surface-emitting light-emitting diodes, IEEE Photon. Technol. Lett., 13, pp. 85-87 (2001)

3. C. Chen, M. Hargis, J. Woodall, E. Yablonovitch, GHz bandwidth GaAs light-emitting diodes, Appl. Phys. Lett., 74, pp. 3140-3142 (1999)

4. A. Bushuev et al, Light emitters for high-speed optoelectronic digital-to-analog conversion, Journal of Physics: Conference Series. IOP Publishing, 1164 (1), 012004 (2019)

5. C. Wu, Design and Layout of Multi GHz Operation of Light Emitting Diodes, CS Mantech Conference, 1, pp. 247-250 (2010)

6. A. Shubert, Temperature and modulation characteristics of resonant-cavity lightemitting diodes, IEEE Journal. Lightwave Techol.,14, 1721 (1996) 
7. S. Laref, S. Mec-abih, B. Abbar, B. Bouhafs, A. Laref, First-principle calculations of electronic and positronic properties of AlGaAs, Physica B: Condensed Matter, 396, pp. 169-176 (2007)

8. A. Schubert, LEDs, FIZMATLIT, 2, 496 (2008)

9. M. Feng, N. Holonyak, Jr., and W. Hafez, Light-emitting transistor: Light emission from InGaP/GaAs heterojunction bipolar transistors, Appl. Phys. Lett., 84, pp. 151-153 (2004)

10. H. W. Then, M. Feng, N. Holonyak, Experimental determination of the effective minority carrier lifetime in the operation of a quantum-well n-p-n heterojunction bipolar lightemitting transistor of varying base quantum-well design and doping, Appl. Phys. Lett., 91, 033505 (2007)

11. C.H. Wu, G. Walter, H.W. Then, M. Feng, and N. Holonyak, Scaling of light emitting transistor for multi-GHz optical bandwidth, Appl. Phys. Lett., 94, pp. 171101-171103 (2009)

12. G. Walter, C.H. Wu, H.W. Then, M. Feng, and N. Holonyak, 4.3 GHz optical bandwidth light emitting transistor, Appl. Phys. Lett., 94, pp. 241101-241103 (2009) 